\title{
Plasma Equilibrium in Self-Sustaining Toroidal Discharge
}

\author{
Shigeo Nagao \\ Engineering School, Chubu University, Kasugai, 487 Japan.
}

(Received October 20, 1987/Revised Manuscript Received March 1, 1988)

\begin{abstract}
It is shown that there exists a plasma equilibrium in a toroidal discharge without the toroidal field, where the plasma current is composed solely of diamagnetic current, driven by the plasma pressure gradient and so is of self-sustaining in principle. This type of configuration seems to suggest a tokamak like device and its corresponding operation for the FRC experiment or to offer a new method of initial current build - up in the tokamak.
\end{abstract}

\section{Keywords :}

self-sustaining, $\mathrm{Z}$ pinch, plasma equilibrium, dipole current density, vertical field, aspect ratio, high beta, stationary fusion reactor,

In the previous works [1],[2], it was concluded that in every toroidal system the dipole current or dipole current component of toroidal discharge is necessary to balance with the hoop force associated with the plasma pressure and especially in the case of tokamak, the necessity of dipole component in the ohmic current for the plasma equilibrium is shown by an exact analysis. In the present note, as the limiting case of vanishing toroidal field in a tokamak, the plasma equilibrium of a toroidal discharge is studied, since the confinement of high beta plasma, the self-sustaining feature of solely diamagnetic discharge current in this configuration and the small aspect ratio of the plasma column are most interesting features as a fusion reactor in future, though some important problems on the plasma stability may still be remained to be solved.

It is easy to understand that the plasma equilibrium in the present configuration with an axial symmetry can be studied in the toroidal coordinate system $(\rho, \theta, s)$ along the circular magnetic axis of radius R, as shown in Fig. 1, by the terms of magnetic field $\vec{B}$ and current density $\vec{j}$, as follows.

$$
\vec{B}=I \vec{u}-\vec{u} \times \nabla \Psi, \quad \vec{u}=\vec{e}_{s} / h, \quad h=1-(\rho / R) \cos \theta
$$

and

$$
\mu_{0} \vec{j}=\operatorname{rot} \vec{B}=-\zeta \Psi \vec{u}-\vec{u} \times \nabla I
$$




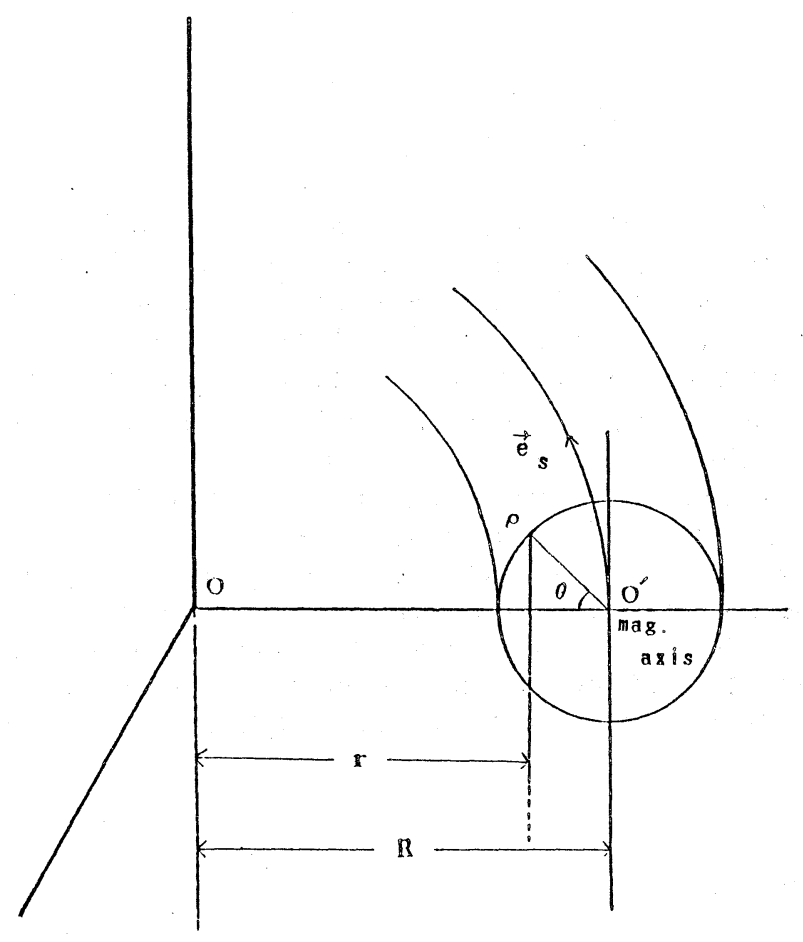

Fig. 1 The toroidal coordinate system $(\rho, \theta, \mathrm{s})$.

Here $\psi$ is the magnetic surface fuction and $\mathrm{P}$ and $\mathrm{I}$ are the plasma pressure and the toroidal field respectively, both being assumed as function of $\psi$. The operator $\zeta$ is given by

$$
\zeta=\frac{\partial^{2}}{\partial \rho^{2}}+\frac{1 \partial}{h \rho \partial \rho}+\frac{\partial^{2}}{\rho^{2} \partial \theta^{2}}-\frac{k \sin \theta \partial}{h \rho \partial \theta}
$$

Then from the equation of plasma equilibrium $\nabla P=J \times \vec{B}$, we obtain the Grad-Shafranov equation, given by

$$
\zeta \Psi+\mu_{0} h^{2} \frac{d P}{d \Psi}+I \frac{d I}{d \Psi}=0
$$


and

$$
\mu_{0} \vec{j}=-\zeta \Psi \vec{u}-\vec{u} \times \nabla I=\mu_{0} h \frac{d P}{d \Psi} \vec{e}_{s}+\frac{d I}{d \Psi} \vec{B}
$$

In the present case of the toroidal discharge with null toroidal field, i.d. $I=0$, the plasma current $\vec{j}$ of eq. (5) becomes solely toroidal and is self-sustaining as mentioned above, since it is composed of the diamagnetic current driven by the pressure gradient. However it should be mentioned that the plasma current becomes purely toroidal if $\mathrm{dI} / \mathrm{d} \psi=0$, even though the field $B$ is finite, as is in the case of a tokamak. Since in the build-up phase of toroidal field in the tokamak, the poloidal current induced in the plasma can have the same direction with the diamagnetic current and so the condition $\mathrm{dI} / \mathrm{d} \psi=0$ may be relatively easy to be realized. Thus both cases of $B=0$ case of toroidal discharge and $\mathrm{dl} / \mathrm{d} \psi=0$ case of tokamak build-up phase have the same current and pressure distribution and therefore the same magnetic surface function. This suggests that such a toroidal discharge may offer an interesting new method of initial current build-up for the tokamak experiments.

Now it will be permitted to assume for simplicity the pressure profile to be given as follows.

$$
P=P_{0}\left(1-\Psi / \Psi_{a}\right)^{3}
$$

where $\psi_{a}$ is the value on the plasma boundary. Eq. (6) is a simplest form which makes the current vanish continuously on the boundary.

Then it is easy to obtain the magnetic surface function $\psi$ by solving the Grad-Shafranov equation eq. (4), that is,

$$
\begin{aligned}
\Psi= & \left(\sqrt{3 \mu_{0} P_{0}} / 2\langle a\rangle\right)\left[\rho^{2}+\varepsilon \rho^{2} \cos 2 \theta-\{(5+\varepsilon) / 4 R\} \rho^{3} \cos \theta\right. \\
& \left.+(Q / R) \rho^{3} \cos 3 \theta+\cdots \cdots \cdots\right]
\end{aligned}
$$

Here $\varepsilon$ and $Q$ are the ellipticity and triangularity of the magnetic surface respectively. When the $\varepsilon$ is large enough, the elongated magnetic surface becomes similar to that of field reversal configuration plasma [3]. However the magnetic surface function looks just like that of a tokamak but the beta value is here $100 \%$. It is to be noted that the shift of magnetic axis by the plasma pressure, called Shafranov shift in a tokamak, is not observed in eq. (7). The annular poloidal field coils arranged around the plasma column to shape the magnetic surface $(\varepsilon$ and $Q)$ might be expected to make an important contribution to the plasma stability, as in the case of a tokamak. Now the toroidal current density is written by 


$$
\mu_{0} j=[1-(\rho / R) \cos \theta]\left(\sqrt{12 \mu_{0} P_{0}} /\langle a\rangle\right)\left(1-\Psi / \Psi_{a}\right)^{2}
$$

and the dipole component of plasma current density is necessary for the balance with the hoop force, associated with the plasma pressure of annular shape and the vertical field $B_{V}$ without the beta term should be applied for the balance only with the hoop force of ring current, as is reported in Ref. [1], that is

$$
B_{V}=-\frac{\mu_{0} J}{4 \pi R}\left[\ln (8 R /\langle a\rangle)+\ell_{i} / 2-2\right]
$$

The product of total particle number $\mathrm{N}\left[10^{20} / \mathrm{m}\right]$ and temperature $\mathrm{kT}[\mathrm{KeV}]$ of the confined plasma can be roughly estimated with the total discharge current $\mathrm{J}[\mathrm{MA}]$, as follows.

$$
N k T=4.68 J^{2}\left[10^{20} / \mathrm{m}\right] \cdot[\mathrm{keV}]
$$

It need not to say that these values of the above example have to be submitted to the stability criterion, which may be improvable by further studies in detail, especially by the toroidal curvature effect, the shaping of magnetic surface by the poloidal field coils and the control of the current profile by RF or NBI power.

Fig. 2 shows some examples of magnetic surfaces and current profile on the equator plane of the torus. Here the expansion of eq. (7) is performed up to the sixth order of $(\rho / R)$ power. It is remarkable that in order to have elongated magnetic surfaces, it is necessary to apply no small amount of negative triangular $Q$ component of poloidal field, as well as the elliptic $\varepsilon$ component.

In conclusion it is shown that there exists a plasma equilibrium of a toroidal discharge without the toroidal field, where the plasma current is composed solely of diamagnetic current, driven by the plasma pressure gradient and so is of self-sustaining in principle. This type of field configuration seems to suggest a tokamak like device and its corrensponding operation for the FRC experiment or to offer a new method of initial current build-up in the tokamak.

\section{ACKNOWLEDGEMENTS}

This work has been supported partly by the Special Research-Aid Funds of Chubu University and partly by the Grant in-Aid from the Japanese Ministry of Education, Science and Culture. 

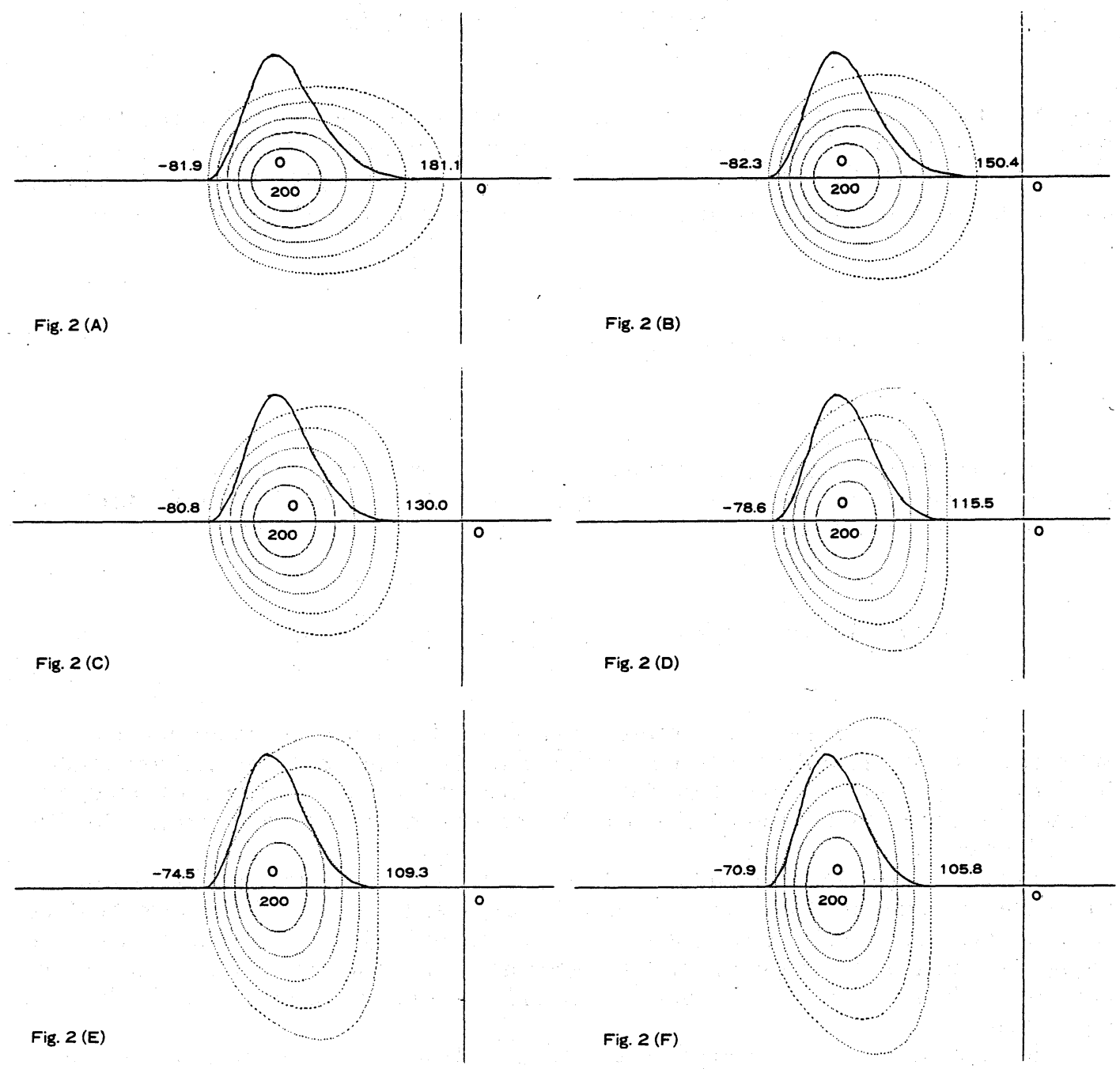

Fig. 2 Examples of magnetic surfaces and plasma current profiles.

$\mathrm{R}=2.00 \mathrm{~m},\langle\mathrm{a}\rangle=80 \mathrm{~cm}$,

$\mathrm{j}=2.44 \mathrm{MA} / \mathrm{m}^{2}$ and $\mathrm{J} \simeq 1.64 \mathrm{MA} .\langle\mathrm{n} \mathrm{kT}\rangle \simeq 6.26\left[10^{20} / \mathrm{m}^{3}\right] \cdot[\mathrm{keV}]$

$\begin{array}{ll}\text { (A) } \varepsilon=0.0 \text { and } \mathrm{Q}=0.0 & \text { (B) } \varepsilon=0.1 \text { and } \mathrm{Q}=0.0\end{array}$

$\begin{array}{ll}\text { (C) } \varepsilon=0.2 \text { and } \mathrm{Q}=0.0 & \text { (D) } \varepsilon=0.3 \text { and } \mathrm{Q}=0.0\end{array}$

(E) $\varepsilon=0.4$ and $\mathrm{Q}=-0.1(\mathrm{~F}) \varepsilon=0.5$ and $\mathrm{Q}=-0.2$

\section{REFERENCES}

1) S. Nagao : Energy. Independence Conference (Fusion Energy and Plasma Physics), Aug. 17-21, 1987. Rio de Janeiro, Brasil, 18C-11-4.

2) S. Nagao : $\ulcorner$ Role of Dipole Current in the Tokamak Equilibrium」 to be published elsewhere in near future.

3) H. Itoh : Journ. Phys. Soc. Japan, Vol. 50, No. 6 (1981) p. 2109. 